\title{
Investigation of the Lateral Reversible Region of YBCO Bulk above a Permanent Magnet Guideway
}

\author{
Yujie Qin · Yunxing Shu
}

Received: 23 September 2012 / Accepted: 30 November 2012 / Published online: 15 December 2012

(C) The Author(s) 2012. This article is published with open access at Springerlink.com

\begin{abstract}
The lateral reversible region of the high- $T_{c}$ superconductor (HTSC) bulk YBCO above a permanent magnet guideway (PMG) is investigated experimentally in this study. The dependence of the lateral reversible region upon selected parameters, such as Field Cooling Height (FH), Working Height (WH), and magnetic field structure, is studied. Results show that the lateral reversible region of the HTSC bulk is not only proportional to the magnitude of the guidance force or guide-force hysteresis, but closely related to the external applied magnetic field structure, $\mathrm{FH}, \mathrm{WH}$, and the size and shape of the HTSC bulk. A conclusion that the lateral reversible region of an HTSC bulk over the single peak symmetrical PMG prior to multipeak Halbach PMG is drawn. The results may be helpful to the design and optimization of maglev systems composed of a permanent magnet guideway and HTSCs.
\end{abstract}

Keywords YBCO bulk $\cdot$ Time relaxation $\cdot \mathrm{NdFeB}$ guideway $\cdot$ Change

\section{Introduction}

After the stable levitation of a $\mathrm{NdFeB}$ permanent magnet above a high- $T_{c}$ superconductor (HTSC) bulk YBCO sample immersed in liquid nitrogen was observed by Hellman et al. in 1988 [1]; the stability contributed to the intrinsic feature of HTSCs with strong flux pinning made many applications [2-5] possible. For a maglev system that is composed of a permanent magnet guideway (PMG) and HTSCs,

\section{Y. Qin $(\bowtie) \cdot$ Y. Shu}

Department of Computer and Information Engineering, Luoyang Institute of Science and Technology, Luoyang, Henan 471023, People's Republic of China

e-mail: qyjswjtu@vip.sohu.com some studies have been reported on experimental, theoretical, and/or computational aspects of the lateral restoring force or guidance force [6-13]. The lateral stability of the high- $T_{c}$ superconducting (HTS) maglev vehicle system is related to the lateral reversible region and, therefore, the study of the lateral reversible region of the bulk HTSC above a permanent magnet guideway (PMG) is very important for understanding the system. However, the reports on this topic were few after the related studies [14, 15].

It is well known that a wider lateral reversible region means better lateral stability and larger available lateral force (we also call this the guide force in a maglev vehicle system). In this paper, we study the dependence of the lateral reversible region upon Field Cooling Height $(\mathrm{FH})$, Working Height (WH) under two different types of magnetic field structure, i.e., monopole peak symmetrical PMG and multipeak Halbach PMG.

\section{Experiment and Results}

The three melting textured growth YBCO HTS bulks used in this study have different parameters; the parameters and photographs of the bulks are shown in Table 1 and Fig. 3. The two types of PMGs supply different applied fields, and their structures and magnetic distributions are shown

Table 1 Parameters of HTS bulks employed in the experiment

\begin{tabular}{llllll}
\hline Shape & $\begin{array}{l}\text { Length } \\
(\mathrm{mm})\end{array}$ & $\begin{array}{l}\text { Width } \\
(\mathrm{mm})\end{array}$ & $\begin{array}{l}\text { Height } \\
(\mathrm{mm})\end{array}$ & $\begin{array}{l}\text { Diameter } \\
(\mathrm{mm})\end{array}$ & $\begin{array}{l}\text { Number } \\
\text { of seeds }\end{array}$ \\
\hline Cylindrical & - & - & 18 & 30 & 1 \\
Cylindrical & - & - & 12.5 & 50 & 1 \\
Rectangular & 64 & 32 & 13 & - & 3 \\
\hline
\end{tabular}




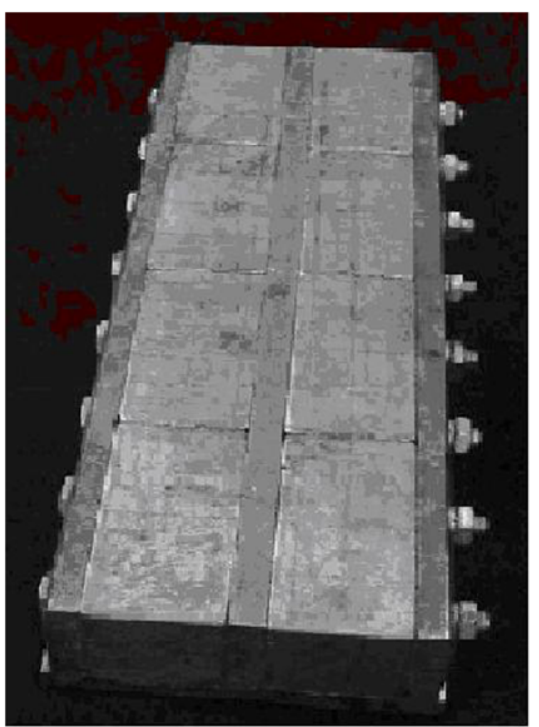

(a)

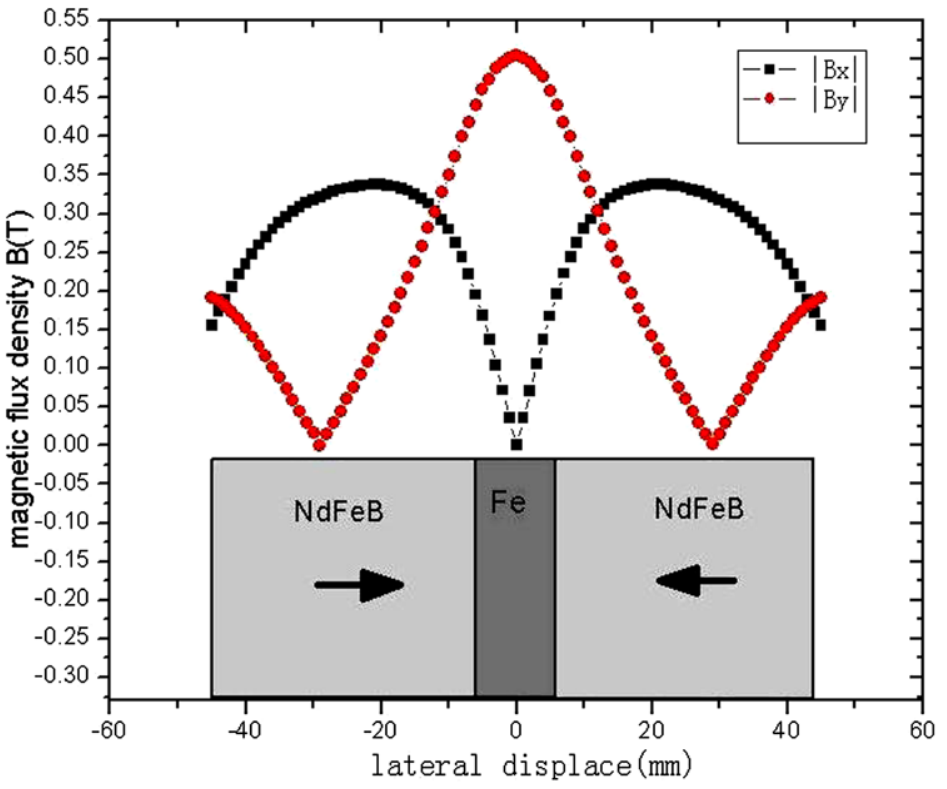

(b)

Fig. 1 Monopole peak PMG (a) photograph (b) magnetic field distribution of the PMG

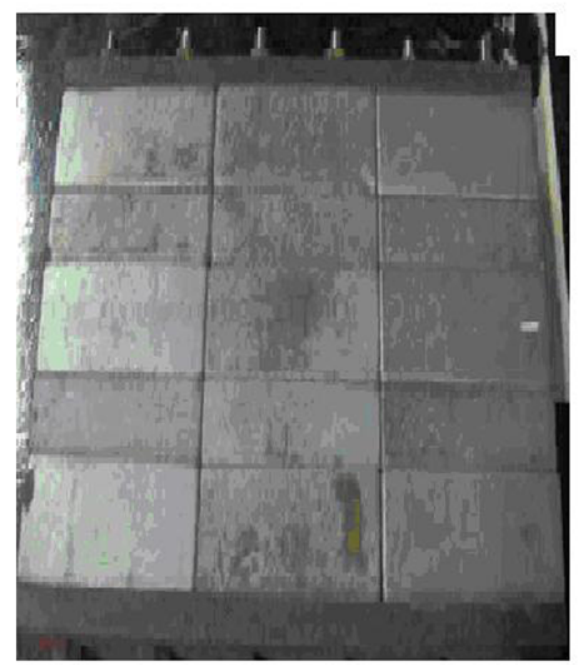

(a)

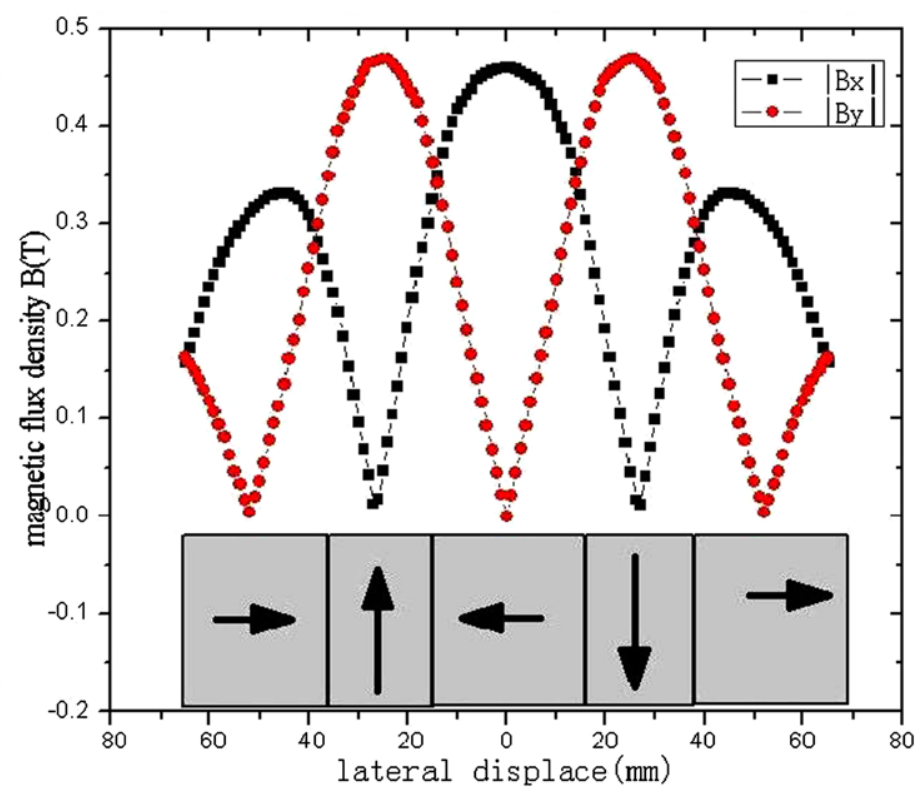

(b)

Fig. 2 Multipole Halbach structure (a) photograph (b) magnetic field distribution of the PMG

in Figs. 1 and 2. The SCML-02 measurement system, described elsewhere [16], was employed to obtain information required for this study. The experimental schematic diagram and coordinate of the HTS maglev system employed in the work are shown in Fig. 4.

The experimental procedures used to investigate the lateral reversible region of $\mathrm{YBCO}$ bulk above a permanent magnet guideway are as follows. The bulk YBCO was first immersed in liquid nitrogen at a certain $\mathrm{FH}$ above the PMG (distance between the bottom of the sample and the surface of the PMG) for 10 minutes to make sure the sample had transitioned to the superconducting state under field-cooling conditions. Then the bulk YBCO was lowered to a particular working height $(\mathrm{WH})$ to measure the guidance force using the SCML-02 measurement system. In the test case, the bulk YBCO was moved in a horizontal loop from $x=0$ to a 
Fig. 3 Photograph of HTS

bulks in the experiment

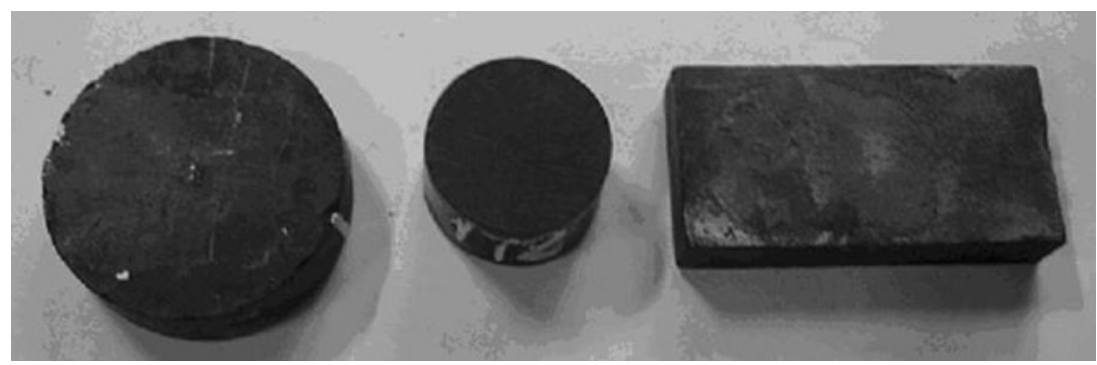

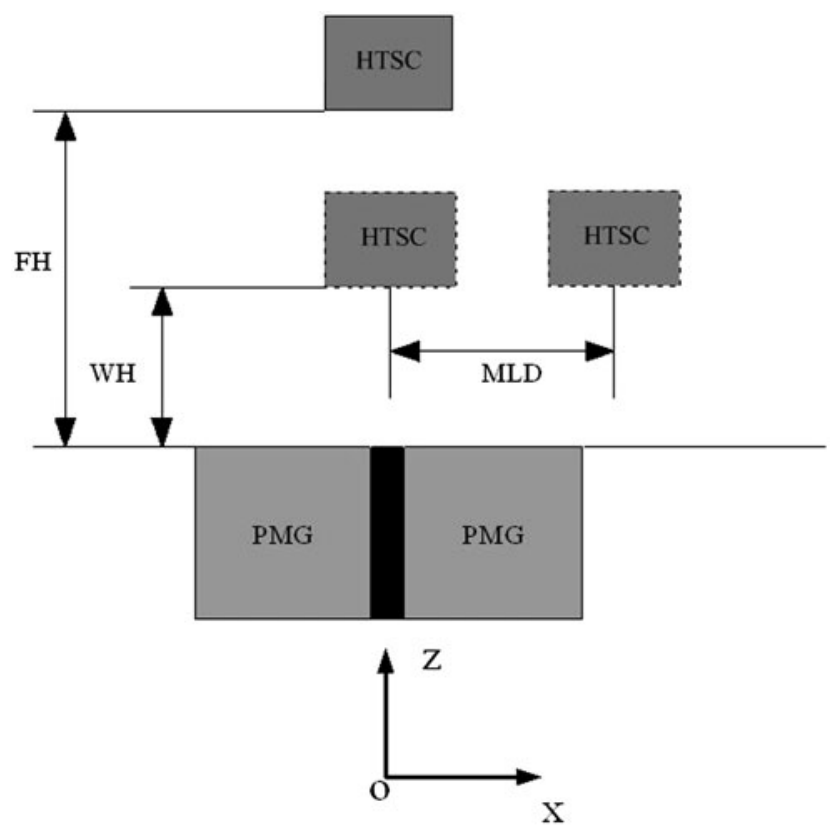

Fig. 4 The experimental schematic diagram and coordinate of the HTS maglev system

given offset position (we called this offset displacement the maximum lateral displacement (MLD)). Figure 5 shows the guidance force exerted by the bulk HTSC with a diameter of $30 \mathrm{~mm}$, which was subjected to the monopole peak PMG magnetic field under the test condition of MLD $=10 \mathrm{~mm}$ and $\mathrm{FH}=\mathrm{WH}=5 \mathrm{~mm}$, respectively.

As shown in Fig. 5, the guidance forces increase rapidly on the curved OA segment and are opposite to the lateral displacement direction when the bulk HTS departs from the initial position $\mathrm{O}$. It shows that the guidance force prevents the bulk from departing the primary equilibrium point to maintain the lateral stability. During the course of the bulk's returning to the initial position after the bulk moves to the MLD, the guidance force decreases with decreasing lateral displacement on curve $\mathrm{AB}$ and the direction of the guidance force is also negative. That is helpful for the bulk's return to the initial position. While the direction of the guidance force turns to positive from negative in accordance with the direction of lateral displacement when the bulk passes point B, the guidance force will prevent the bulk from returning to the initial position. In order to enable the bulk to return to

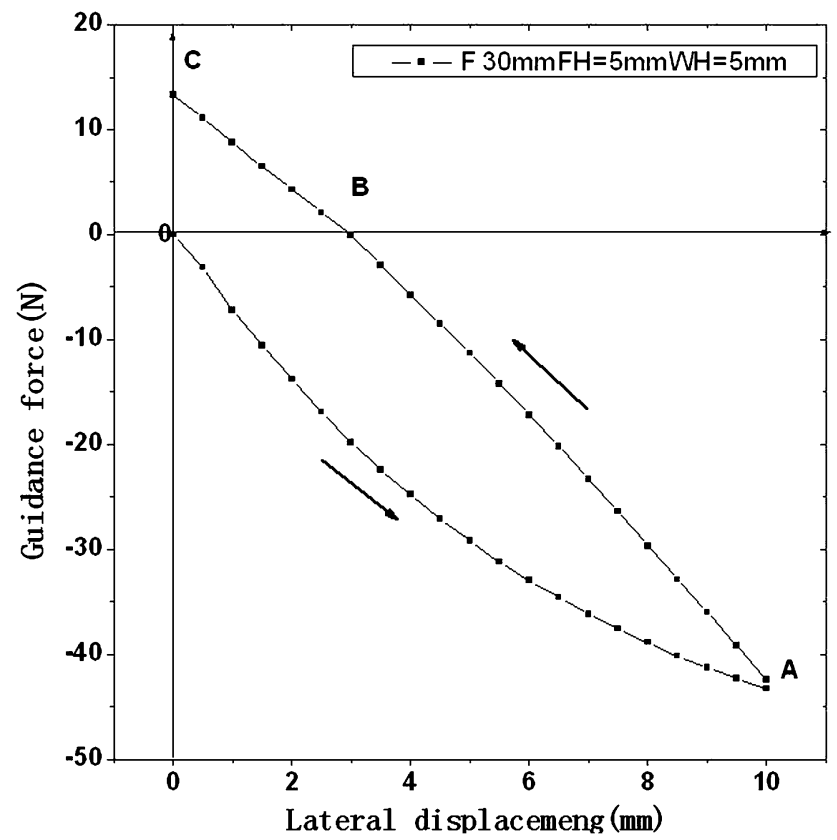

Fig. 5 Measurement of lateral reversible region of the bulk HTS over the monopole peak PMG

the initial position, an external force that equals the magnitude of the force at point $\mathrm{C}$ will be applied, otherwise the bulk will stay in position $\mathrm{B}$. Here, $\mathrm{OB}$ is defined as the lateral nonreversible displacement to describe the lateral reversible region. Less OB means a wider lateral reversible region and better lateral stability.

In order to investigate the lateral reversible region of YBCO bulk above a permanent magnet guideway, the similar loops were repeated under different conditions that involved magnetic field structure (a monopole peak PMG and a Halbach PMG), working condition, size, and shape of the bulk HTS. The FC and WH were chosen to approximate the actual operational situation of the HTS maglev vehicle. Only the experiments in a positive $x$-axis direction were done, because the system had the translation symmetry; they have the same results in the negative $x$-axis direction.

The experimental results are shown from Figs. 6, 7, 8 and 10. In these experiments, we first investigate the lateral reversible region of bulk HTS over a monopole peak PMG. For the lateral reversible region over a Halbach PMG, the re- 


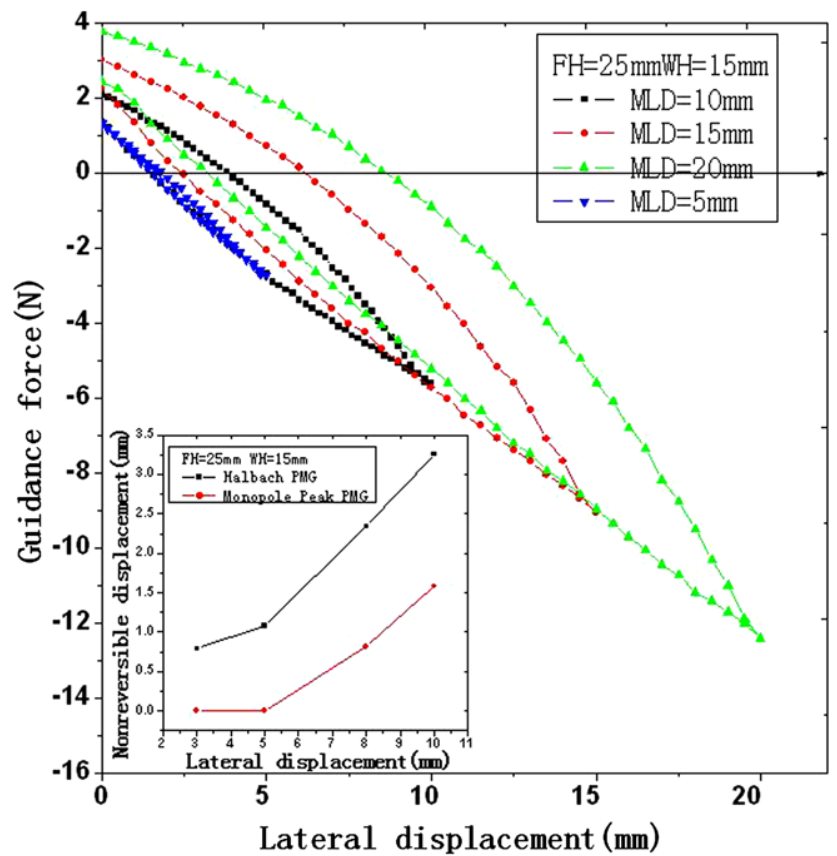

Fig. 6 The lateral reversible region under the condition of invariable $\mathrm{FH}$ and $\mathrm{WH}$ and variable maximum lateral displacement (MLD)

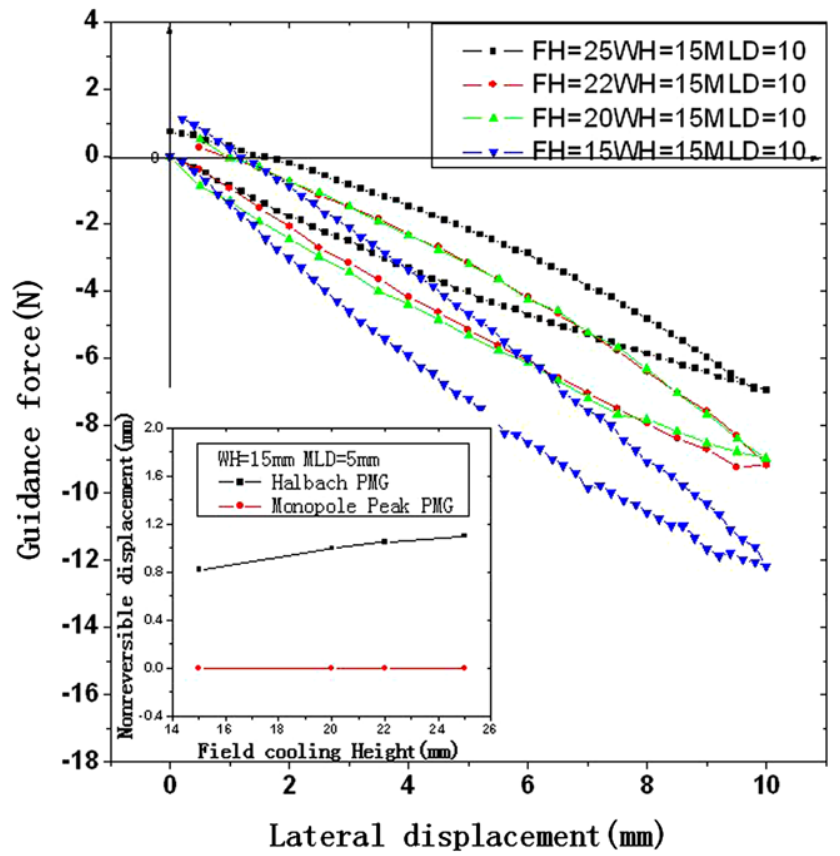

Fig. 7 The lateral reversible region under the condition of invariable WH and MLD, variable FH

sults show that the varied tendency of the nonreversible displacement is in accordance with the monopole peak PMG under the conditions mentioned above. We only provide curves compared with the monopole peak PMG under different conditions, shown in an interleaved pattern. Figure 6 shows the lateral reversible region under the condition of

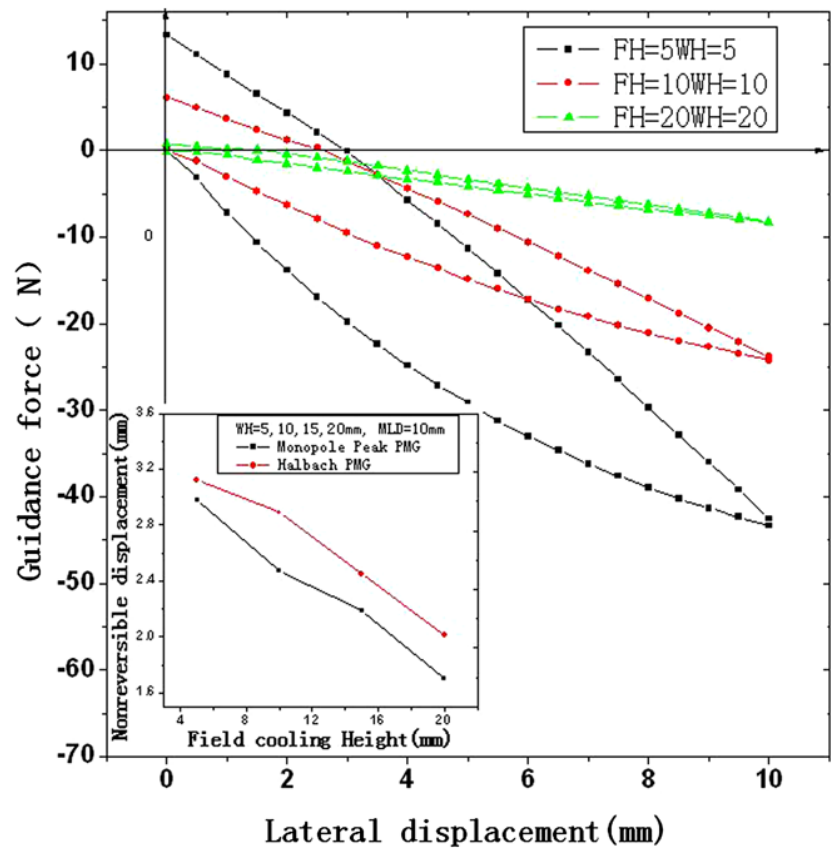

Fig. 8 The lateral reversible region under the condition of invariable MLD, variable FH and WH

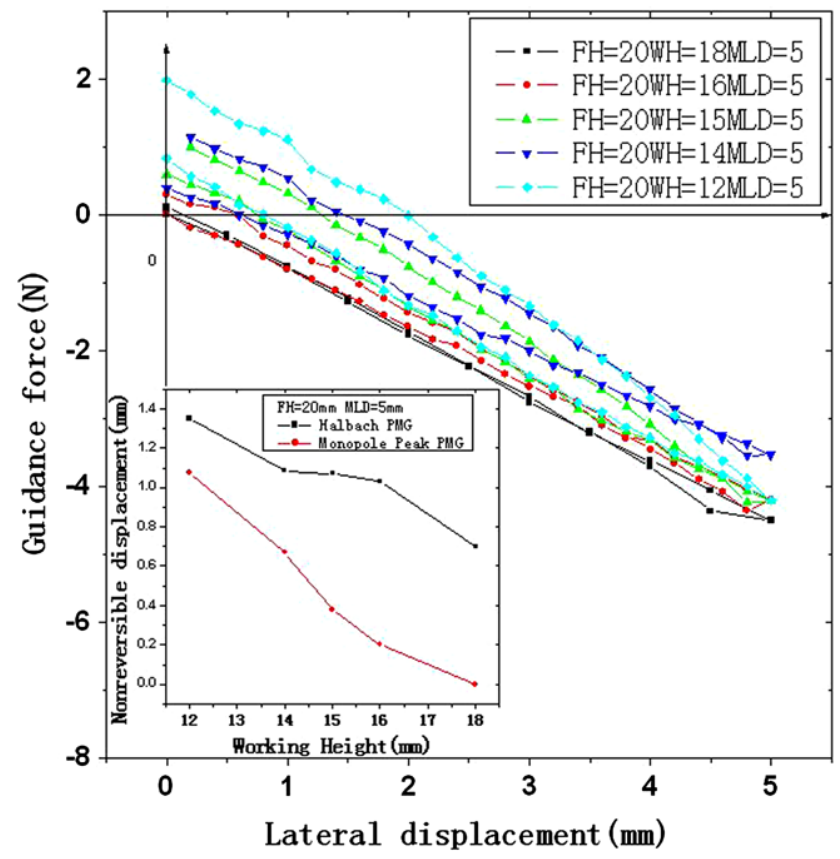

Fig. 9 The lateral reversible region under the condition of invariable $\mathrm{FH}$ and LD, variable WH

invariable $\mathrm{FH}$ and $\mathrm{WH}$ and variable maximum lateral displacement (MLD). As Fig. 6 shows, when the MLD is larger than $5 \mathrm{~mm}$, the test loop is nearly nonreversible. The irreversibility of the lateral motion increases with the increment of the MLD. Figure 7 shows the lateral reversible region under the condition of constant WH and MLD, variable FH. 


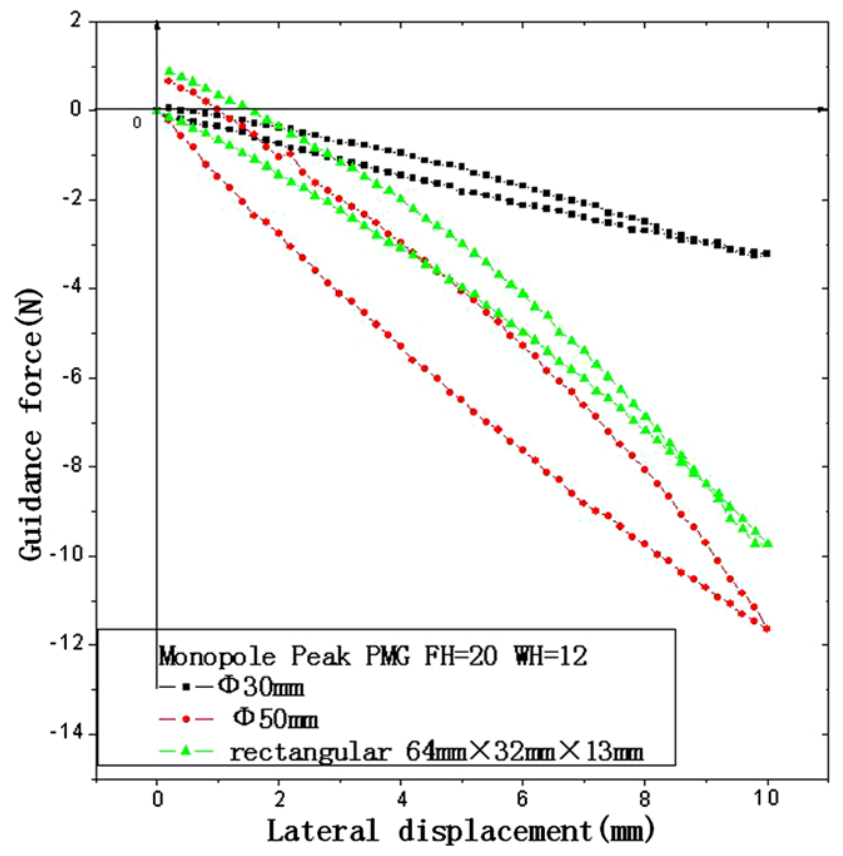

Fig. 10 The dependence of the lateral reversible region upon size, shape, seeds number of bulk over the monopole peak PMG

From Fig. 7, we can see that the irreversibility of the lateral motion increases very slowly with the increment $\mathrm{FH}$. Figures 8 and 9 show the lateral reversible region under the condition of constant MLD, variable FH, WH and constant FH, LD, variable WH, respectively. From Figs. 8 and 9, we know the irreversibility of the lateral motion decreases with the increment FH and WH. However, the nonreversible displacement of HTS bulk over the Halbach PMG is larger than that of the monopole peak PMG under the three cases mentioned above. Figure 10 shows the dependence of the lateral reversible region upon size, shape, and seeds number of bulk over the monopole peak PMG. As Fig. 10 shows, the lateral reversible region is relevant with their properties.

\section{Analysis and Discussion}

As shown in Fig. 6, in the case of constant FH and WH, the nonreversible displacements of the bulk HTS subjected to different maximum lateral displacements are different and the nonreversible displacements increase with increasing MLD. For infinite length PMG ignoring ending effect, the magnetic field is composed of horizontal component $B_{x}$ and vertical component $B_{z}$. The magnetic forces of the bulk HTS over PMG come from the following equation based on magnetostatics $[17,18]$ :

$\vec{F}=\int_{s c}(M \cdot \nabla)_{(x, z)} d v$
From Eq. (1), the guidance force of the bulk YBCO can be written as

$G=F_{x}=l \int_{0}^{h} d z \int_{-w}^{w} M \frac{\partial B_{z}}{\partial x} d x$

where the parameters $l, h, w$ represent the length (or equivalent length), height, and half-width (or equivalent width) of the bulk YBCO. and

$M=\mu_{s} B_{z}$

The parameter $\mu_{s}$ is the permeability constant of the bulk YBCO.

Define $f(x)$ as

$f(x)=\frac{B_{z}(x)-B_{z}(x-d)}{d}$

When the bulk moves distance $d$ along the horizontal direction, the variation of $B_{z}$ and $\mathrm{M}$ can be expressed by $f(x)$ as follows:

$\Delta B_{z}=B_{z}(x-d)-B_{z}(x)=-f(x) d$

$\Delta M=\mu_{s} \Delta B_{z}=-\mu_{s} f(x) d$

Differential expression (2) is represented by differential expressions (5) and (6), the guidance fore of the bulk experienced during lateral displacement can be written as

$G=l \int_{0}^{h} d z\left(\int_{-w}^{0} \Delta M_{1} f(x) d x+\int_{0}^{w} \Delta M_{2} f(x) d x\right)$

After the field cooling process, $B_{z}$ in the bulk is equals to that of the applied field, and no guidance force exists under this condition. When the bulk YBCO moves horizontally, the applied field changes and variation of $B_{z}$ occurs. According to Eqs. (6) and (7), variations of $M$ will generate the guidance force. From Eq. (5), the sign of $\Delta M$ always opposite to $f(x)$, so the guidance force always prevents the bulk from departing the original equilibrium point. When the lateral displacement is small, we can regard that the magnetic flux does not move and dislocate, so the bulk will return to the initial equilibrium point. In this case, nonreversible displacement will be zero and no remnant appears in the bulk. When the lateral displacement is larger, the magnetic flux dislocates from its initial pinning position by the flux flow and creep motion, then the remnant $M$ occurs in the bulk after the whole lateral motion. It causes energy loss so that the bulk will not return to the initial equilibrium position and the nonreversible displacement is not equal to zero, and the nonreversible displacement increases with increasing lateral displacement. For the two different types of magnetic field structure, as shown Figs. 1 and 2, variation of $B_{z}$ (by in Figs. 1 and 2) over the multi-peak Halbach along horizontal direction is fast than that of the monopole peak PMG, so that the influence of the flow and creep motion upon the lateral motion is obvious for the HTS bulk over the multipeak Halbach. This is the reason that the nonreversible displacement 
of HTS bulk over the Halbach PMG is larger than that of the monopole peak PMG.

From Figs. 7 and 8, we can see that the nonreversible displacement of the bulk decreases with decreasing FH at constant $\mathrm{WH}$, while the nonreversible displacement decreases with increasing $\mathrm{FH}$ at variable $\mathrm{WH}$. As the hysteresis of the guidance force is decided mainly by $\mathrm{WH}$, the hysteresis of guidance force is almost the same at constant $\mathrm{WH}$ but variable FH. The nonreversible displacement of the bulk depends on the magnitude of the guidance force. Larger guidance force generates when the bulk trapped more magnetic flux at lower FH. So, the nonreversible displacement of the bulk decreases with decreasing FH at constant WH. For variable $\mathrm{FH}$ and $\mathrm{WH}$, the nonreversible displacement of the bulk is influenced by magnitude and hysteresis of the guidance force simultaneously but mainly by hysteresis. Magnetic flux of the bulk trapped increases with decreasing $\mathrm{FH}$ and $\mathrm{WH}$, which causes the enlargement of magnitude and hysteresis of the guidance force but hysteresis is more obvious. As a result, the nonreversible displacement decreases with increasing $\mathrm{FH}$ at variable $\mathrm{WH}$.

From Fig. 9, we can see that the nonreversible displacement of the bulk increases with decreasing WH at constant FH. The hysteresis of the bulk increases with decreasing $\mathrm{WH}$, and it causes enlargement of the nonreversible displacement.

From the interleaved pattern of the Figs. 6 to 9, we can see that the nonreversible displacement of HTS bulk over the monopole peak symmetrical PMG is larger than that of multipeak Halbach PMG. It means the lateral reversibility of the HTS bulk over the monopole peak PMG is better than that of the Halbach PMG. This mainly depends on the magnetic field structure.

\section{Conclusions}

With regard to two types of PMGs, the lateral reversible region of the HTS bulk has been investigated experimentally in this study. Relevant theory based on magnetostatics is used to analyze the magnetization process and the guidance force. From the experimental results and the ensuing analyses, we may think that the remnant magnetization caused by the flux dislocation is the essential source of the lateral irreversibility. From the results, we see that the lateral reversible region is not only proportional to the magnitude of the guidance force or guide-force hysteresis, but closely related to the external applied magnetic field structure, FH, WH, and the size and shape of the HTS bulk. From the point, we may draw a conclusion that the lateral reversible region of an HTSC bulk over monopole peak symmetrical PMG prior to multipeak Halbach PMG.
Open Access This article is distributed under the terms of the Creative Commons Attribution License which permits any use, distribution, and reproduction in any medium, provided the original author(s) and the source are credited.

\section{References}

1. Hellman, F., Gyorgy, E.M., Johnson, D.W. Jr., et al.: Levitation of a magnet over a flat type II superconductor. J. Appl. Phys. 63(2), 447-450 (1988)

2. Weh, H., et al.: High- $T_{c}$ superconductors calculation model and possible Maglev applications. In: Proc. 14th Int. Conf. on Magnetically Levitated Systems, Germany, pp. 217-222 (1995)

3. Hull, J.: Superconducting bearings supercond. Sci. Technol. 13(2), R1-R15 (2000)

4. Yang, W.J., Qiu, M., Liu, Y., Wen, Z., et al.: Levitation characteristics in an HTS Maglev launch assist test vehicle. Supercond. Sci. Technol. 20(3), 281-286 (2007)

5. Strasik, M., Johnson, P.E., et al.: Design, fabrication, and test of a $5-\mathrm{kWh} / 100-\mathrm{kWh}$ flywheel energy storage utilizing a high temperature superconducting bearing. IEEE Trans. Appl. Supercond. 17(2), 2133-2137 (2007)

6. Johansen, T.H., Yang, Z.J., Bratsberg, H., et al.: Lateral force on a magnet placed above a planar $\mathrm{YBa}_{2} \mathrm{Cu}_{3} \mathrm{O}_{x}$ superconductor. Appl. Phys. Lett. 58(2), 179-181 (1991)

7. Johansen, T.H., Mestl, H., et al.: Investigation of the lateral magnetic force and stiffness between a high- $T_{c}$ superconductor and magnet of rectangular shapes. J. Appl. Phys. 75(3), 1667-1670 (1993)

8. Yang, Z.J., Hull, J.R., et al.: Amplitude and frequency dependence of hysteresis loss in a magnet-superconductor levitation system. J. Appl. Phys. 78(3), 2097-2100 (1995)

9. Mora, J., Carrera, M., et al.: Flux trapping and levitation forces in directionally solidified superconducting $\mathrm{YBa}_{2} \mathrm{Cu}_{3} \mathrm{O}_{7}$ ingots. J. Appl. Phys. 79(8), 6596-6598 (1996)

10. Hikihara, T., Isozumi, G.: Modeling of lateral force-displacement hysteresis caused by local flux pinning. Physica C 270(1-2), 6874 (1996)

11. Hull, J.R., Cansiz, A.: Vertical and lateral forces between a permanent magnet and a high-temperature superconductor. J. Appl. Phys. 86(11), 6396-6404 (1999)

12. Sanagawa, Y., Ueda, H., et al.: Characteristics of lift and restoring force in HTS bulk. IEEE Trans. Appl. Supercond. 11(1), 1797$1800(2001)$

13. Wang, J., et al.: Guidance forces on high temperature superconducting Maglev test vehicle. IEEE Trans. Appl. Supercond. 13(2), 2154-2156 (2003)

14. Wang, X.R., Ren, Z.Y., et al.: Guidance force in an infinitely long superconductor and permanent magnetic guideway system. Supercond. Sci. Technol. 18, S99-S104 (2005)

15. Liu, W., et al.: A method to increase the lateral reversible region of bulk YBCO above a permanent magnet guideway. J. Low Temp. Phys. 158(5-6), 922-933 (2010)

16. Wang, S.Y., Wang, J.S., Deng, C.Y., He, Q., et al.: Laboratoryscale high temperature superconducting Maglev launch system. IEEE Trans. Appl. Supercond. 17(2), 2091-2094 (2007)

17. Johansen, T.H., Bratsberg, H.: Theory for lateral stability and magnetic stiffness in a high- $T_{c}$-superconductor-magnet levitation system. J. Appl. Phys. 74(6), 4060-4065 (1993)

18. Davis, L.C.: Lateral restoring force on a magnet levitated above a superconductor. J. Appl. Phys. 67(5), 2631-2636 (1990) 